\title{
COMPARISON OF MUSCLE ACTIVATION AND BARBELL KINEMATICS DURING BENCH PRESS WITH DIFFERENT LOADS
}

\author{
Roland VAN Den TillaAR ${ }^{1}$, Colby Sousa ${ }^{2}$ \\ ${ }^{1}$ Nord University, Department of Sport Science and Physical Education, Levanger, \\ Norway \\ ${ }^{2}$ Sports Performance Research Institute New Zealand, AUT, Auckland, New Zealand
}

\begin{abstract}
The aim of this study was to compare barbell kinematics and muscle patterning in bench press with different loads, but with maximum effort, in young males with resistance training experience. Ten healthy experiences strength-training males (aged $27.3 \pm 5.9$ years, body mass $82.8 \pm 16.6 \mathrm{~kg}$, height $1.78 \pm 0.05 \mathrm{~m}$, experience $7.3 \pm 4.2$ years) performed maximal effort bench presses (1-2 repetitions) with loads varying from $30 \%$, with $10 \%$ increments until 100\% of 1-RM. Muscle activity of seven muscles and barbell kinematics were measured during descending and ascending phases. Average and peak upwards lifting velocity increased, while lifting time decreases with each decreasing load. In general, the maximal activation of most muscles decreases with decreasing loads, but it was not linear. No effect of loads was shown for the biceps brachii and posterior deltoid muscles. Based upon these findings, it was concluded that maximal lifting velocity may compensate for increased loads, which may allow resistancetrained males who are both healthy or in rehabilitation to avoid heavy loads but experience similar muscle activation. By decreasing the loads, the mechanical stress decreases and time to recover is reduced. Using lower loads with maximal lifting velocity may allow athletes to increase the total volume without increasing the risk of injuries.
\end{abstract}

Key words: electromyography, EMG, resistance exercise, lower body strength 


\section{INTRODUCTION}

Bench press is a popular exercise utilized to elicit muscular adaptations of the upper body such as strength, hypertrophy and power. With variations in load and intensity, lifting kinematics and neuromuscular adaptations will also express differences $[13,14]$. Therefore, based on the current goals of a resistance training program, appropriate load selection can be made to accomplish these outcomes. Specifically, heavier loads ( $>80 \%$ of 1RM) have traditionally been thought to be necessary to use in order to recruit fasttwitch motor units according to the size principle [14, 19], whereas lighter loads $(<60 \%)$ were thought to produce adaptations of training speed specificity and enhance mechanical power output [10, 14]. Ballistic movements (muscle contractions at maximum velocities over a short period of time) are typically performed with lower loads and high effort which may lead to a lower recruitment threshold; thus, recruiting high-threshold motor units [20]. This has been investigated in previous studies where peak and average velocity decreases with increasing external load [17, 18]. Additionally, with different stimuli being placed on the body (i.e. different rep ranges, intensities and states of fatigue), variability will be seen in joint and barbell kinematics $[4,21,25]$. However, the literature is scarce when examining neuromuscular activity and kinematics in relationship to different loads while being instructed to lift at maximal intended velocity.

The principle of specificity states that training should be relevant and appropriate to the sport which the individual is training for in order to optimize results. Specifically, in resistance training, developing a consistent and efficient movement pattern is desirable; therefore, it seems pertinent to know how the kinematics of an exercise change with a change in training variables. To maximize efficiency in the bench press, maximal effort should be exerted throughout the concentric portion of the lift to ensure its success and to ensure maximal strength gains [12]. In a multiple repetition set to volitional failure with $75 \%$ of $1 \mathrm{RM}$ in the bench press, time to complete the concentric portion of the lift more than doubled with a concomitant decrease in the peak and mean concentric velocity [5]. As the subjects progressed from first to last repetition, the lifting kinematics became more like those of the 1RM [5]. This may give insight to the importance of training intensity and the idea that if volume and intensity are equated, rep ranges are not as important. Additionally, this may provide implications for mimicking strength training without utilizing heavier loads which is desirable for certain populations (i.e. injured or elderly). However, it is possible that the subjects did not truly produce maximal effort during each repetition as instructions to do so were not given. 
The understanding of both external (kinematics) and internal (bioelectric activity of muscles) characteristics of an exercise provide valuable information about the athlete. Male subjects performed both the eccentric and concentric portion of the lift and concentric only bench press throws using loads of $15 \%, 30 \%, 45 \%, 60 \%, 75 \%, 90 \%$ and $100 \%$ of their previously determined 1RM bench press. Analysis of electromyography (EMG) data indicated that the peak level of activity and the averages over the concentric movement were reduced at lower loads [15]. However, this data in bench throws does not necessarily carry over to traditional bench press; thus, further research is warranted. During examination of EMG activity in the flat bench press, single repetitions were performed with 70, 80, 90 and $100 \%$ of $1 \mathrm{RM}$. As load increased, muscle activity varied greatly and in a non-linear fashion in the pectoralis major, anterior deltoid, triceps brachii, and latissimus dorsi [11]. However, subjects were not instructed to complete each lift with maximal intent which could have an influence on muscle activity and velocity.

Therefore, the purpose of this study is to compare muscle activation patterning and barbell kinematics in bench press with different loads in experienced resistance-trained athletes instructed to lift with maximal effort on the concentric portion of the lift. We hypothesize that muscle activity of the measured muscles will increase only after $60 \%$ of 1-RM (size-principle) and a linear decreased velocity in both downward and upward phases.

\section{MATERIALS AND METHODS}

\section{Subjects}

Ten healthy males experienced with resistance training were recruited (aged 27.3 \pm 5.9 years, body mass $82.8 \pm 16.6 \mathrm{~kg}$, height $1.78 \pm 0.05 \mathrm{~m}$, experience $7.3 \pm 4.2$ years). Inclusion criteria were being able to lift their own body weight $(103 \pm 16.5 \mathrm{~kg})$ in $1-\mathrm{RM}$ bench press and no injuries or pain that could reduce their maximal performance. None of the participants were competitive powerlifters or weightlifters. The participants did not conduct any resistance training of the legs 72 hours before testing. Each participant was informed of the testing procedures and possible risks, and written consent was obtained prior to the study. The study complied with the current ethical regulations for research and approved by the Norwegian Centre for Research Data, in conformance with the latest revision of the Declaration of Helsinki. 


\section{Procedures}

The participants were tested in two occasions; in the first occasion to establish their 1-RM and the second occasion to test muscle activity and performance with loads varying from $30 \%$ to $100 \%$ of 1 -RM. At each occasion the participants started with a standardized, progressive, specific warm-up protocol according to van den Tillaar, et al. [21]. After a general warm-up or 5 minutes jogging on a treadmill, the protocol consisted of 15 repetitions at $30 \%, 10$ repetitions at $50 \%$, and 6 repetitions at $80 \%$ of the participants' self-reported 6-RM loads in bench press. The bench press was performed in a power rack (Gym 2000, Modum, Norway) with an Olympic barbell (diameter $=2.8 \mathrm{~cm}$, length $=1.92 \mathrm{~m})$. During the test protocol, the head, shoulders and hips were supported by the bench with a $\sim 90^{\circ}$ flexion in the knees. Each participant chose an optimal grip and feet position, which were controlled for during the whole test. Two spotters assisted the participants in the preload phase by lifting and stabilizing the barbell until the participant had fully extended arms. The barbell was lowered in a controlled manner, lightly touched the chest and lifted back with maximal effort to the starting position with fully extended elbows. No bouncing of the barbell or to raise the lower back from the bench was allowed. The participants were instructed to use a self-selected tempo in which they had full control over their lifting technique and performed with maximal effort.

At the first occasion after the final warm-up set, the load was increased to approximately $95 \%$ of the participants' self-reported 1-RM. The load was then increased by $2.5-5.0 \mathrm{~kg}$ until failure. Failure was defined by the following criteria: 1) the participants failed to complete a lift, 2) the participants could not complete the lift with proper technique, or 3) both the participant and the test leader agreed that the participant would not be able to lift $2.5 \mathrm{~kg}$ more. The 1-RM was achieved within 2-4 attempts. Each attempt was separated by a pause of $4-5$ minutes.

At the second visit after the final warm-up set, the participant starting testing using the different loads. The loads began from $30 \%$, with $10 \%$ increments until $100 \%$ of 1-RM, which was based on 1-RM achieved by each participant. Importantly, the participants were instructed to accelerate the loads in the entire concentric movement, which resulted in a bench press throw using the lowest loads (i.e. $30 \%-50 \%$ of $1-\mathrm{RM}$ ). Two experienced spotters ensured that the barbell did not land back on their body. The different loads were randomized for each participant, with random order determined by a random number generator. Two repetitions per load from $30 \%$ to $60 \%$ were conducted, while from $70 \%$ to $100 \%, 1$ repetition per load was performed. A rest of 3-5 minutes was given between each attempt [7]. 


\section{Measurements}

Wireless electromyography (EMG) was recorded with a sampling frequency of $1000 \mathrm{~Hz}$ by using a Musclelab 6000 system and analyzed by Musclelab v10.5.67 software (Ergotest Technology AS, Langesund, Norway). The skin was shaved, abraded, and washed with alcohol before placing the gel-coated self-adhesive electrodes (Dri-Stick Silver circular sEMG Electrodes AE-131, NeuroDyne Medical, USA). The electrodes (11 mm contact diameter and $2 \mathrm{~cm}$ center-to-center distance) were placed along the presumed direction of the underlying muscle fiber according to the recommendations by SENIAM $[9,16]$. The electrodes were placed on the right side on the muscle belly of the biceps brachii, lateral and long head of triceps brachii, sterncostal and clavicular part of the pectoralis and anterior and posterior deltoid muscles. To minimize noise from the surroundings, the raw EMG signal was amplified and filtered using a preamplifier located close to the sampling point. The EMG signals were converted to root mean square (RMS) EMG signals using a hardware circuit network (frequency response $20-500 \mathrm{kHz}$, averaging constant $100 \mathrm{~ms}$, total error $\pm 0.5 \%$ ). The mean RMS EMG signals of each muscle during the descending and ascending phases of the lift with each load were used for further analysis. The beginning, lowest point and end of each lift were identified by using a linear encoder (ET-Enc-02, Ergotest Technology AS, Langesund, Norway) attached at the inside of the weights to the barbell. The encoder measures the upward phase duration of the barbell with a resolution of $0.075 \mathrm{~mm}$ and counts the pulses with 10-ms intervals [2]. Total descending and ascending times and distances were measured. Peak and average velocity of the barbell during the descending and ascending phases were calculated by using a 5-point differential filter with Musclelab v10.73 software (Ergotest Technology AS, Langesund, Norway).

\section{Statistical Analysis}

To assess the differences in EMG activity during the upward phase of the different loaded bench presses, a two-way analysis of variance (ANOVA) 2 (descend, ascend phase) $\times 8$ (percentage of 1-RM: 30-100) with repeated measures was used. Furthermore, a one-way ANOVA with repeated measures (percentage of 1-RM) was used to identify barbell kinematics and EMG activity per phase. If significant differences were found, a Holm-Bonferroni post-hoc test was performed. In cases where the sphericity assumption was violated, the Greenhouse-Geisser adjustments of the $p$-values were reported. The level of significance was set at $p \leq 0.05$. When $p$ was between 0.05 and 0.10 it was indicated with a trend [1]. Statistical analysis was performed with 
SPSS version 24.0 (SPSS Inc, Chicago, IL). Effect size was evaluated with $\eta^{2}$ (Eta squared) where $0.01<\eta^{2}<0.06$ constitutes a small effect, a medium effect when $0.06<\eta^{2}<0.14$, and a large effect when $\eta^{2}>0.14$ [3].

\section{RESULTS}

The average and peak barbell velocity in both phases changed significantly over lifted loads $\left(\mathrm{F} \geq 4.2, p \leq 0.047 ; \eta^{2} \geq 0.32\right.$ ). Post-hoc comparison showed that average and peak ascending lifting velocity increased with each decrease in lifting load (Figure 1), while in the descending phase only peak velocity increased from 100 to $60 \%$ and for 60 to $30 \%$ of $1-\mathrm{RM}$. The average velocity in descending phase only increased significantly from 100 to $50 \%$ and from 60 to $30 \%$ of 1-RM (Fig. 1). Also, an effect of load was found for descending and ascending lifting time $\left(\mathrm{F} \geq 4.3, p \leq 0.024 ; \eta^{2} \geq 0.33\right)$ and lifting distance $\left(F \geq 5.1, p \leq 0.018 ; \eta^{2} \geq 0.36\right.$ ). Post hoc comparison revealed no significant differences in descending barbell distance and only a significant decrease in descending lifting time from $80 \%$ to $50 \%$ of 1 -RM. In the ascending phase the lifting time increased with every load and the lifting distance from 80 to $50 \%$ and with the 40 and $30 \%$ of $1-\mathrm{RM}$.

A significant effect of lifting phase was found for each muscle ( $\mathrm{F} \geq 9.5$, $p \leq 0.013 ; \eta^{2} \geq 0.51$ ), with more EMG activity in the ascending than the descending phase, except of the biceps brachii that showed the opposite (Figure 2). Load had a significant effect in both phases upon all muscles $\left(\mathrm{F} \geq 3.7, p \leq 0.026 ; \eta^{2} \geq 0.29\right)$ except the biceps brachii and posterior deltoid $\left(\mathrm{F} \leq 1.1, p \geq 0.39 ; \eta^{2} \leq 0.11\right)$. The post-hoc comparison indicated that with regard to the EMG activity, for the pectoralis, triceps and anterior deltoid muscles, EMG activity decreased from 100 to 90 and/or $80 \%$ of 1-RM in both phases. From $80 \%$ loads the max EMG activity (ascending phase) did not decrease significantly any more for the clavicular part of the pectoralis major, while for the sternal part it decreased again from 70 to $40 \%$ of 1-RM. for The anterior deltoid and lateral head of triceps it decreased their activity from 60 to $30 \%$ of 1-RM, while it also decreased between 80 and $60 \%$ and 70 and $50 \%$ for respectively lateral head and medial head of the triceps (Figure 2). 

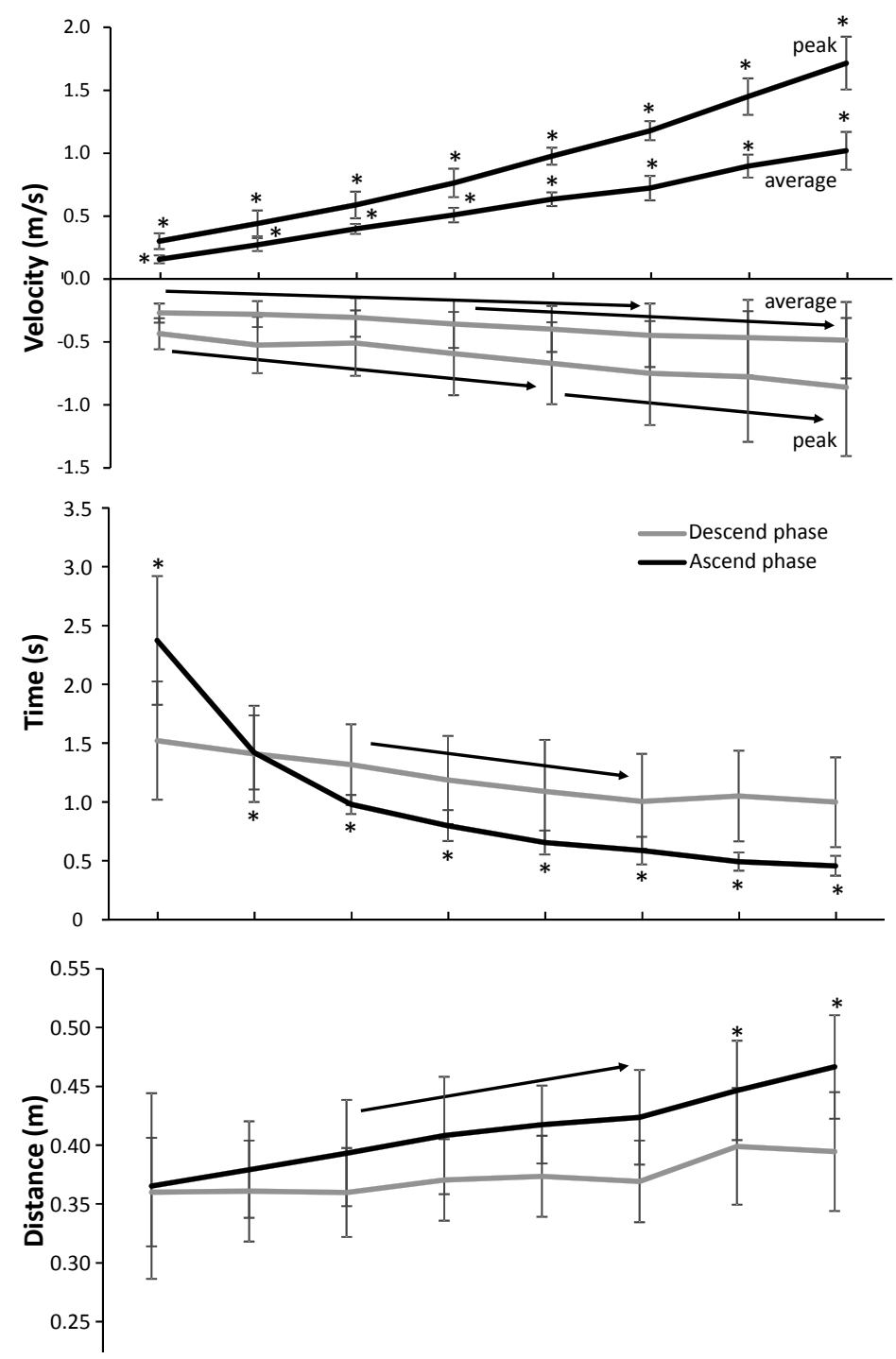

Figure 1. Mean (SD) average and peak velocity, lifting time and distance during descending and ascending phase at different percentages of 1-RM (30\%-100\%) of bench press. * indicates a significant difference $(p \leq 0.05)$ with all other percentages.

$\rightarrow$ indicates a significant difference $(p \leq 0.05)$ between this percentage and all percentages away from the sign. 


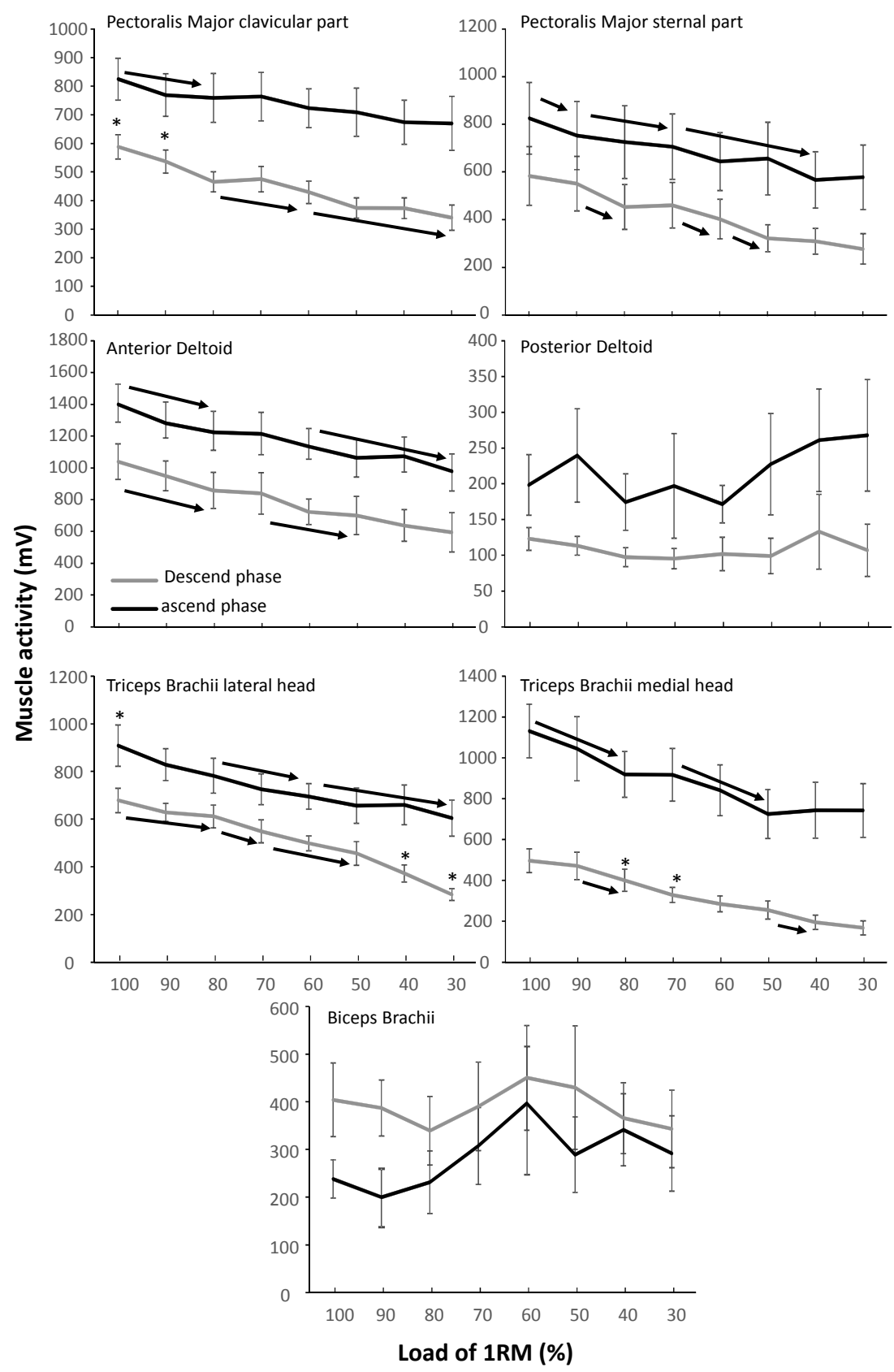

Figure 2. Mean (SEM) root mean square (RMS) EMG activity for each percentage of 1-RM during descend and ascending phase in of the biceps brachii, lateral and long head of triceps brachii, sterncostal and clavicular part of the pectoralis and anterior and posterior deltoid muscles during bench press.

$\rightarrow$ indicates a significant difference $(p \leq 0.05)$ between this percentage and all percentages away from the sign.

* indicates a significant difference $(p \leq 0.05)$ with all other percentages. 


\section{DISCUSSION}

The aim of this study was to compare barbell kinematics and muscle patterning in bench press with different loads, but with maximum effort, in young males with resistance training experience. Average and peak upwards lifting velocity increased, while lifting time decreases with each decreasing load (Figure 1). In general, the maximal activation of most muscles decreases with decreasing loads, but it was not linear. No effect of loads was shown for the biceps brachii and posterior deltoid muscles.

In support of our hypothesis and by previous studies, as load decreased, average and peak concentric velocity significantly increased and the concentric duration of the lift significantly decreased $[17,18]$. This was to be expected due to the laws of Newton $(\mathrm{F}=\mathrm{mx}$ ) with the acceleration equaling the force divided by the weights lifted. As load increases and maximal force stays relatively the same due to instructions to lift at maximal intended velocity each repetition, acceleration is likely to decrease as load increases. With greater loads, the acceleration was lower in the beginning, resulting in lower velocity and longer upward phase duration, which eventually resulted in the peak velocity appearing earlier in the movement with increasing loads $[15,23]$. The shift to an early peak in vertical velocity may be due to an attempt to enter the sticking region with the greatest possible velocity, thereby increasing the likelihood of completing the lift [4]. Furthermore, with heavy load (> 85\% 1RM) or fatigue, the sticking region occurred, which causes a longer upward phase duration $[11,24]$.

Greater downward distance in the $30 \%$ and $40 \%$ were the only significant changes in distance. This may be because subjects were instructed to lift at maximal intended velocity which resulted in bench throws with these lower loads. Additionally, since the load was so light, subjects may have not approached the lift with the same mindset as they would have with a heavier load. This may have led to less scapular retraction which could have inevitably increase the distance the bar would need to travel. To support this idea, there was less distance recorded on the downward phase the closer to the 1RM the subjects got which is also supported by Duffey and Challis [4]. As the load gets heavier, there is a greater emphasis on technique primarily to stay safe, but also to be as biomechanically efficient as can be in order to complete the lift.

Increasing muscle activation was observed with increasing loads but was not linear. These results are true for both the eccentric and concentric portions of the exercise. These results agree with [11] where the greater the weight on the barbell, the greater the muscle activity of shoulder muscles 
was. In addition, at 90 and $100 \%$ of $1 \mathrm{RM}$, greatest muscle activity for the lateral head of the triceps was measured. Logically, this makes sense as the more difficult a lift is, the more muscle recruitment is required in order to complete the lift. It is important to note that these EMG values were recorded during non-fatiguing contractions ( 1 and 2 rep sets in the current study) since fatigue may cause EMG patterns to be altered [6]. Though subjects only completed one repetition, implications can be made based on previous data. For example, the time to lift the bar more than doubled from the first to last rep causing a decrease in both mean and peak velocity [4]. While Duffey and Challis [4] looked at a multiple repetition set, most of the kinematic variables analyzed became more like those of the maximal lift as the subjects progressed through a set to volitional failure with $75 \%$. In the present study, attempts with 90 and 100\% were deemed at or near maximal and similar results of kinematics variables were found. This gives insight to the concept that as long as volume and effort are equated, similar muscular adaptations can be seen. Furthermore, an adequate stimulus is required to be placed on the muscle in order for an adaptation to be created highlighting the importance of training at high efforts (i.e. 0-4 reps shy of failure). This does not necessarily have to be with low or higher reps but rather, a combination that will allow for the individual to maintain adherence to their current training program while still eliciting adaptation.

For the pectoralis, triceps, and anterior deltoid muscles, EMG activity decreased from 100 to 90 and/or $80 \%$ of 1RM in both phases. From $80 \%$ loads, the max EMG activity (concentric phase) did not decrease significantly any more for the clavicular part of the pectoralis major, while for the sternal part it decreased again from $70-40 \%$ of $1 \mathrm{RM}$. The difference in EMG activity in the regions of the pectoralis major may be attributed to anatomical differences, grip width, and/or differences in setup on the bench. Additionally, EMG activity of the biceps brachii and posterior deltoid were not influenced by the increases in \%1RM. This may be due to the synergistic relationship between these muscles. Specifically, the pectoralis, anterior deltoid and triceps are acting as the mobilizers to complete the movement (hence the increased activity), while the biceps brachii and posterior deltoid are acting as stabilizers leading to little EMG activity. As the weight increases, a greater demand is placed on these mobilizers in order to sufficiently complete the lift which may explain the results. For the antagonist biceps brachii and posterior deltoid, there were no effects of different load on EMG activity. Additionally, muscle activity in these antagonist muscles did not follow any trends with the increases in load. These findings are in contradiction to what was found by van den Tillaar, et al. [22] in the back 
squat where the antagonist biceps femoris and semitendinosus had higher muscle activation using loads above $70 \% 1 \mathrm{RM}$ as a potential result of cocontraction to stabilize the knee and pelvis. These results were not mimicked in the current study potentially as a result of the total musculature used in the bench press being less than that of the back squat. Since the back squat requires more musculature used, co-contraction may be more important in order to remain technically efficient to produce the most force while not getting injured.

To our knowledge, this is one of the few studies examining muscle activation and bar kinematics with increasing loads in the bench press where the participants were instructed to lift at maximal intended velocity. In comparison, peak level of EMG activity and the averages over the concentric movement were reduced at lower loads examined bar kinematics and muscle activation during bench press throws [15] which agrees with the current study. This may be explained by the idea that when throwing heavier loads, the movement is slower and requires greater force production with a longer duration of activation; thus, a greater proportion of the motor unit pool is recruited with the use of both fast and slow twitch motor units [15]. While the standard bench press is different than a bench throw, the idea of moving the barbell at maximal intended velocity may elicit similar changes in both muscle activation and barbell kinematics. Muscle activation for even the lightest loads were large and may be a result of the high accelerations being produced by a ballistic throw suggesting a much higher overload on the neuromuscular system than from a traditional bench press [15]. This has also been shown to be true for explosive squat jumps performed with relatively light loads [8]; therefore, may be an explanation for non-linear muscle activation and barbell kinematics across increasing intensities.

The current study is not without limitations. First, we only used resistance trained males making it difficult to extrapolate findings to other populations. Additionally, only the bench press was analyzed; thus, it is not known if the current results would hold true in single-joint exercises or in multiple repetitions sets while being told to move with maximal intended velocity. Furthermore, there is always a risk of cross talk from nearby muscles using surface EMG, which could generate inaccurate measurements. Finally, the study did not include measurements of peak or angle velocity of the shoulder, elbow or wrist, and no analysis was performed on different parts on the concentric phase, which could demonstrate different technique testing with the different loads.

Based upon the findings of the present study, it was concluded that average and peak upwards lifting velocity increased, while lifting time decreases 
with each decreasing load. In general, the maximal activation of most muscles decreases with decreasing loads, but this was not linear, except not the biceps brachii and posterior deltoid muscles where no effect of loads were shown. This means that maximal lifting velocity may compensate for increased loads, which may allow resistance-trained males who are both healthy or in rehabilitation to avoid heavy loads but experience similar muscle activation. By decreasing the loads, the mechanical stress decreases and time to recover is reduced. Using lower loads with maximal lifting velocity may allow athletes to increase the total volume without increasing the risk of injuries.

\section{ACKNOWLEDGEMENT}

The authors would like to thank the participants. The study was conducted without any funding from companies, manufacturers, or outside organizations.

\section{REFERENCES}

1. Bangalore S, Messerli FH. (2006) Of Statistical Significance: “Trends” Toward Significance and Optimism Bias. J Am Coll Cardiol, 48: 1471.

https://doi.org/10.1016/j.jacc.2006.07.011

2. Bosquet L, Porta-Benach J, Blais J. (2010) Validity of a commercial linear encoder to estimate bench press $1 \mathrm{RM}$ from the force-velocity relationship. J Sports Sci Med, 9: 459-463.

3. Cohen J. (1988) Statistical power analysis for the behavioral sciences. Hillsdale, NJ, England: Lawrence Erlbaum Associates.

4. Duffey MJ, Challis JH. (2007) Fatigue effects on bar kinematics during the bench press. J Strength Cond Res, 21: 556-560.

https://doi.org/10.1519/00124278-200705000-00046

5. Duffey MJ, Challis JH. (2007) Fatigue effects on bar kinematics during the bench press. J Strength Cond Res, 21: 556-560.

https://doi.org/10.1519/00124278-200705000-00046

6. Glass SC, Armstrong T. (1997) Electromyographical activity of the pectoralis muscle during incline and decline bench presses. J Strength Cond Res, 11: 163-167. https://doi.org/10.1519/00124278-199708000-00006

7. Goodman CA, Pearce AJ, Nicholes CJ, Gatt BM, Fairweather IH. (2008) No difference in 1RM strength and muscle activation during the barbell chest press on a stable and unstable surface. J Strength Cond Res, 22: 88-94.

https://doi.org/10.1519/JSC.0b013e31815ef6b3 
8. Hakkinen K, Komi PV, Kauhanen H. (1986) Electromyographic and force production characteristics of leg extensor muscles of elite weight lifters during isometric, concentric, and various stretch-shortening cycle exercises. Int J Sports Med, 7: 144-151. https://doi.org/10.1055/s-2008-1025752

9. Hermens HJ, Freriks B, Disselhorst-Klug C, Rau G. (2000) Development of recommendations for SEMG sensors and sensor placement procedures. J Electromyogr Kinesiol, 10: 361-374. https://doi.org/10.1016/S1050-6411(00)00027-4

10. Kaneko M, Fuchimoto T, Toji H, Suei K. (1983) training effect of different loads on the force-velocity relationship and mechanical power output in human muscle. Scand J Sports Sci, 5: 50-55.

11. Krol H, Golas A, Sobota G. (2010) Complex analysis of movement in evaluation of flat bench press performance. Acta Bioeng Biomech, 12: 93-98.

12. Lander JE, Bates BT, Sawhill JA, Hamill J. (1985) A comparison between freeweight and isokinetic bench pressing. Med Sci Sports Exerc, 17: 344-353. https://doi.org/10.1249/00005768-198506000-00008

13. McBride JM, Larkin TR, Dayne AM, Haines TL, Kirby TJ. (2010) Effect of absolute and relative loading on muscle activity during stable and unstable squatting. Int J Sports Physiol Perf, 5: 177-183. https://doi.org/10.1123/ijspp.5.2.177

14. McBride JM, Triplett-McBride T, Davie A, Newton RU. (2002) The effect of heavy- vs. light-load jump squats on the development of strength, power, and speed. J Strength Cond Res, 16: 75-82.

https://doi.org/10.1519/00124278-200202000-00011

15. Newton RU, Murphy AJ, Humphries BJ, Wilson GJ, Kreamer WJ, Häkkinen K. (1997) Influence of load and stretch shortening cycle on the kinematics, kinetics and muscle activation that occurs during explosive upper-body movements. Eur J Appl Physiol, 50: 311-320. https://doi.org/10.1007/s004210050169

16. Saeterbakken AH, Fimland MS. (2013) Muscle force output and electromyographic activity in squats with various unstable surfaces. J Strength Cond Res, 27: 130-136. https://doi.org/10.1519/JSC.0b013e3182541d43

17. Sanchez-Medina L, Gonzalez-Badillo JJ. (2010) Movement velocity as a measure of loading intensity in resistance training. Int J Sports Med, 31: 347-352. https://doi.org/10.1055/s-0030-1248333

18. Sanchez Medina L. (2010) Movement velocity as a determinant of the level of effort in resistance training, in: PhD Thesis. Sevilla: Universidad Pablo de Olivive.

19. Schmidtbleicher D, Haralambie G. (1981) Changes in contractile properties of muscle after strength training in man. Eur J Appl Physiol Occup Physiol, 46: 221-228. https://doi.org/10.1007/BF00423398

20. Suchomel TJ, Nimphius S, Bellon CR, Stone MH. (2018) The Importance of Muscular Strength: Training Considerations. Sports Med, 48: 765-785. https://doi.org/10.1007/s40279-018-0862-z 
21. van den Tillaar R, Andersen V, Saeterbakken A. (2014) Comparison of muscle activation and performance during $6 \mathrm{RM}$, two legged free-weight squats. Kinesiol Slov, 20: 5-16.

22. van den Tillaar R, Andersen V, Saeterbakken AH. (2019) Comparison of muscle activation and kinematics during free-weight back squats with different loads. PLoS One, 14: e0217044. https://doi.org/10.1371/journal.pone.0217044

23. van den Tillaar R, Ettema G. (2009) A comparison of successful and unsuccessful attempts in maximal bench pressing. Med Sci Sports Exerc, 41: 2056-2063. https://doi.org/10.1249/MSS.0b013e3181a8c360

24. van den Tillaar R, Ettema G. (2010) The "sticking period" in a maximum bench press. J Sports Sci, 28: 529-535. https://doi.org/10.1080/02640411003628022

25. van den Tillaar R, Saeterbakken A. (2014) Effect of fatigue upon performance and electromyographic activity in 6-RM bench press. J Hum Kin, 40: 57-65. https://doi.org/10.2478/hukin-2014-0007

\section{Correspondence to:}

Roland van den Tillaar PhD

Department of Sport Sciences and Physical Education

Nord University

Odins veg 23

7603 Levanger

Norway

E-mail: roland.v.tillaar@nord.no

Phone: +47-5767 1883

Fax: +4774112001 